majority of cases of genu valgum treated by splints in our hospital out-patient rooms, sufficient pressure to mould the bones is not applied; and even in those cases in which it is applied, the bandage and strapping very soon loosen, or the limb rotates just a little, and it is no longer exercised. Even with outside irons and leather bands I doubt very much if sufficient pressure is kept up to mould the bones. Only with the most careful padding with wool or some soft material over the inner aspect of the bone where traction outwards is applied, and over the outer malleolus where the splint will press, would the delicate skin of children stand pressure sufficient for this purpose.

Even with a back splint joined to the outside splint, it is very difficult to prevent some rotation outwards of a genu valgum limb to which considerable pressure is applied to draw the knee outwards, and the least rotation outwards at once lessens the pressure. In order to really mould the bones by means of splints and leg irons much pressure must be used after most careful padding, and the patient must be frequently seen to make sure the limb has not twisted at all, and that the pressure is doing no harm. I do not believe that such pressure can be applied in ordinary out-patient practice, and that it can be maintained while the children are walking about is to my mind incredible. To attach a genu valgum limb to a splint so that it is firmly fixed against it is not sufficient to mould it straight, and the probability is that not many hours after the child has been taken home, especially if it is allowed to run about, the bandage or straps no longer even hold the limb firmly againgst the splint. It must require continuously acting traction outwards against the splint to mould the bones. As I have just said, I do not believe a splint can mould the bone straight whilst children are standing or walking about. A force acting in the form of traction outwards at the knee-joint, which if the ends of the bones were free from pressure might straighten out the curve of the leg bones, would be at once counterbalanced by the pressure on the two ends of the bones from the transmitted weight of the body, for this would be a far greater force than the traction outwards against the splint. Indeed, it seems possible that when the patient is walking about, and the traction outwards cannot straighten out the curve, and this traction is only applied at the knee, it may produce a new curve as represented in Diagram 5, B. But I very much doubt if traction sufficient to mould the bones can be applied whilst the child is walking about. If it can be maintained under such conditions it can certainly act whilst the child is at rest, and so the gradual straightening out of the curve causing the genu valgum and the formation of this new curve would go on alternately, the result would be what I have represented in Diagram 5, c-a partial correction of one curve and the

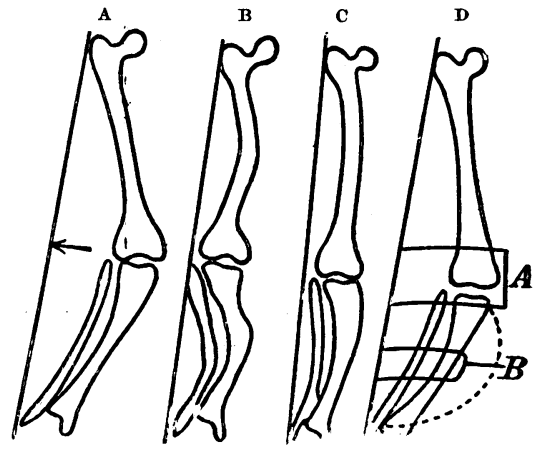

Diagram 5.-A, arrow shows direction of traction outwards towards splint; $\mathbf{B}$, effect if child constantly walking or standing; $\mathbf{C}$, effect splint; $B$, effect if child constantly walking or standing; C, effect of the limbs by.splint ; A, support at knee; B, support for leg.

formation of another. The obliquity outwards of the anklejoint would not be wholly corrected.

I think that the application of an outside splint of iron may. prevent the increase of genu valgum, even if the child runs about, and that the deformity may undergo spontaneous rectification whilst such apparatus are in use just as it may if no splints are employed. But if such support is given only to the knee, by a bandage or strap passing round the splint and the joint, the leg bones may continue to bend below as shown by the dotted line in Diagram 5, D, and therefore I think the shaft of the tibia, and even the lower end of the femur, should be supported also.

This has been much debated, and there is a great difference of opinion with regard to the time when this should be done, and as to what cases require it. Some surgeons never operate below a certain age; but do not agree as to what that age should be. Others only operate when a certain degree of genv valgum is present; but here, again, there is no agreement; and one surgeon operates for a few inches less than another. Again, some surgeons have such a strong faith in the value of splint treatment, you would think from what they say there are no cases splints cannot cure, and that all their patients, even with marked deformity, are content to go on year after year, with very gradual improvement. Mg own feeling $\mathrm{g}$ that in severe cases of genu valgum, even in young childre $n$, if there is reason to think that the stage of softening and yielding of the bones is over, osteotomy may be done, but no doubt the older the child the less the tendency to relapse. Often the parents of children with marked genu valgum are not content to employ splints month after month with a very slow improvement, or perhaps none at all, nor are they willing to keep their children off their feet for months; or, indeed, amongst the poor people, able to do so. They will have their children's legs straightened, even though we point out to them the risk of relapse without the greatest care for years after the operation. In older children, and in young adults in whom the deformity has quite, ceased to increase for some time, and in whom the deformity is marked, I should not hesitate to do osteotomy.

\section{NOTES AND REFERENCES.}

1 BRITISH MEdical JoURNAL, 1897, vol. $i$, p. 1347, 2 This skiagraph is reproduced in my paper in the BRITISH MEDICAL JouRNAL, r898, vol. $i$, p. roo4, Skiagraph No. I. 3 BRITISH MEDICAL JoURNAL, 1897, vol. i, p. 15x4, and vol. ii, p. 53. 4 Loc. cit. 5 Osteotomy, Macewen, 1880. 6 Trans. Royai Academy of Medicine in Ireland, vol. xiv, 1896. p. 409. T BRITISH MEDICAL JoURNAL, 1897, vol.i, p. 1514. 8 Ibid., 1898, vol. i, p. roo3. 9 An illustration of this is given in skiagraph No. $I$ in my paper in the BRITISH MEDICAL JOURNAL, r898, vol. i, p. IOO3. 10 BRITISH MEDICAL JOURNAL, 1897, vol. ii, p. 1638. 11 Ibid., r888, vol. ii, p. 1148.12 Bruns, Beitr. klin. Ch.

\section{SPINA BIFIDA :}

ITS OPERATIVE TREATMENT AMONGST OUT-PATIENTS. BY JAMES H. NICOLL, M.B.,

Extra Surgeon Children's Hospital : Assistant-Surgeon, Western Infirm ary; Consulting Surgeon, Ear Hospital; etc., Glasgow.

ON January inth, 1902, the two following cases were shown at a meeting of the Glaggow Medico-Chirurgical Society.

CASE 1.- G.M., a patient of Dr. Fotheringham, of Motherwell, was brought to the Glasgow Children's Hospital, at the age of 2 months, on August 2nd, 19or. (Fig. ris the reproduction of a photograph taken on that date.) otherwise healthy.

Operation - On August 6 th, four days later, the tumour was excised in operation.- On August The tumour proved to be a pure meningocele.

Progress. - Convalescence was uneventful. The child was nursed throughout by its mother, aided by an aunt, and instructed by the Surgical Sister, who visited the patient daily, dressed the wound on the one occasion on which it was thought advisable, and removed the skin sutures on the eighth day.

now nearly 8 months old. is in perfect health, and was shown to the Society. (Fig 2 is a reproduction of a photograph taken a month previously, that is, some four and a-half months after operation.

CASE II.-B. B., a patient of Dr. Wm. Ross, Glasgow, was brought to the Children's Hospital, at the age of 3 weeks, on November 17 th, 1901. (Fig. 3 is the reproduction of a photograph taken on that date.) The case differed in certain features from the foregoing, and, in demonstrating it to the students, attention was drawn to two points-(a) dimpling of the with and by palpation, and by transmitted light) (b) a considerable degree of paraplegia (evidenced by blueness of feet and legs, absence of voluntary movements of lower limbs, and the repeated development of spasticity on the attempt to excite reflex movement)

Operation.-On November $23 \mathrm{rd}$, a week later, the tumour was excised in the out-patient clinic, and the child thereafter nome in the care of its mother. On opening the sac during the operation there were found four nerve cords coursing over its wall, three on one side and one on the other. Attached to these cords were a number of whitish tumours ranging in size from:that of a pinhead to that of a large pea. The nerve 
cords were dissected from the sac wall, when it was found impossible for lack of space to replace the attached tumours in the spinal canal. The largest of the tumours was therefore detached from the cords and refavial ascir of the patent spinal canal.

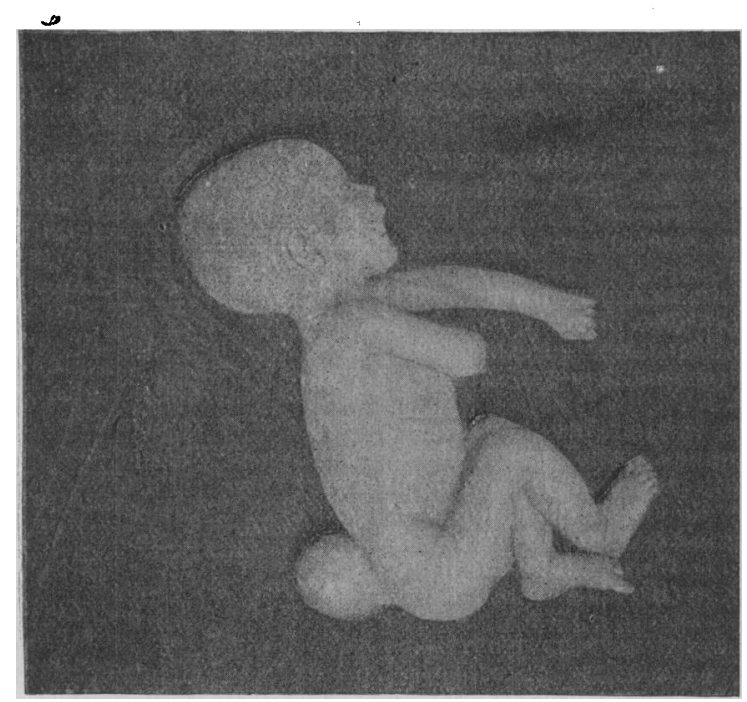

Fig. x. -G. M., aged 2 months.

Progress. - Convalescence, except for temporary carboluria, was uneventful. The child was nursed throughout by its mother and grandmother in chaned the dresings when necessary, and removed the skin sutures the sixtenth day. The child is now, some two months aftor operes on in good health and was shown to the societz. of a photograph taken six weeks after operation.) The flaps have formed

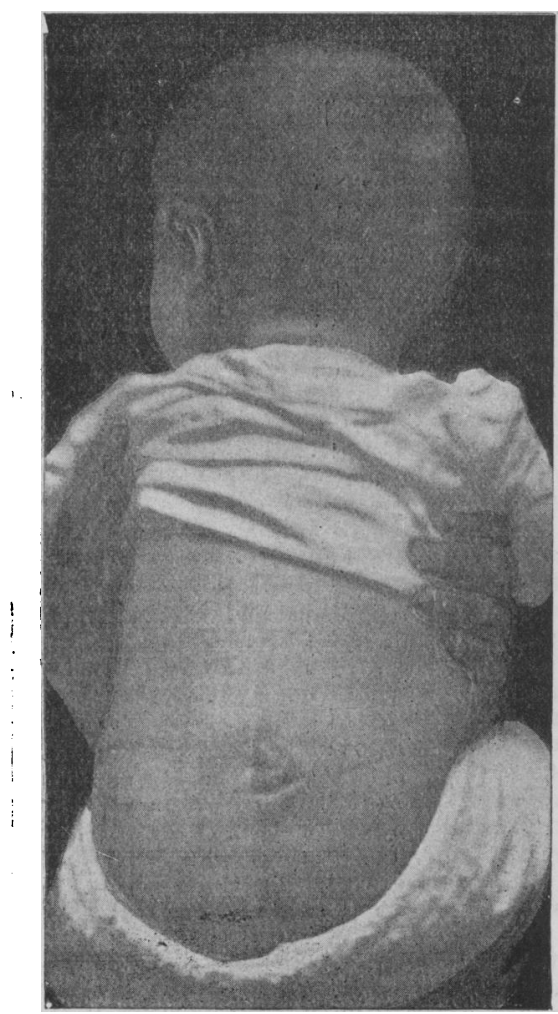

Wig. e. -G. Mo, aged $6 \frac{1}{2}$ months. Photograph taken four and a-half months after excision of the spina bifida. a firm closure of the gap in the posterior wall of the spinal canal. A a firm improvement has taken place in the condition of the lower limbs: Lividity has disappeared. Spasticity has all but entirely subsided, and

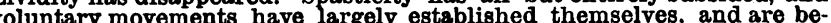
coming daily more perfect.

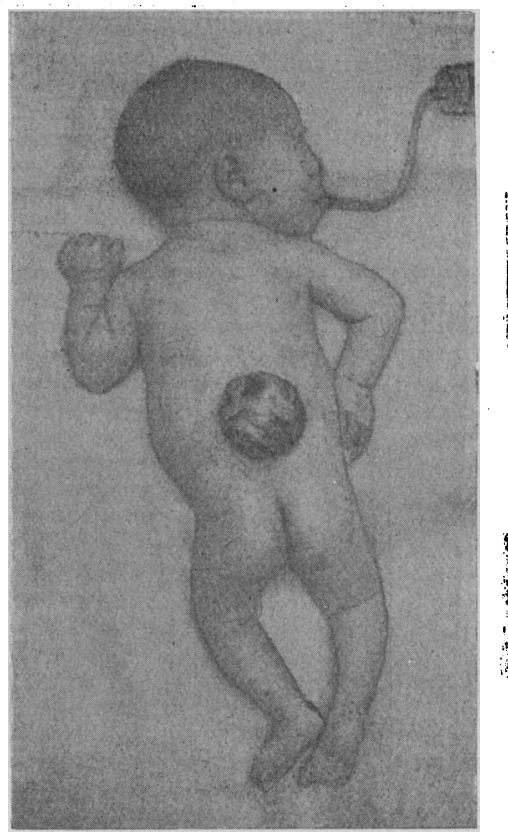

Fig. 3.-B. B., aged 3 weeks.

Fig. 5 is the reproduction of a photograph of the excised sac. It is shrivelled, and its margins incurled, by the action of preservatives. $O$ the portion of its interior exposed, however, are visible several ragged surfaces left after separation of the nerve cords and tumours. Beside the sac are shown three of the largest of these tumours, which histological examination has proved to be of the nature suggested by the naked-eye characters. Dr. Leslie Buchanan, of the West of Scotland Clinical Research Laboratory, has made and stained sections of one of the tumours, and reports it to be a "false (plexiform) neuroma."

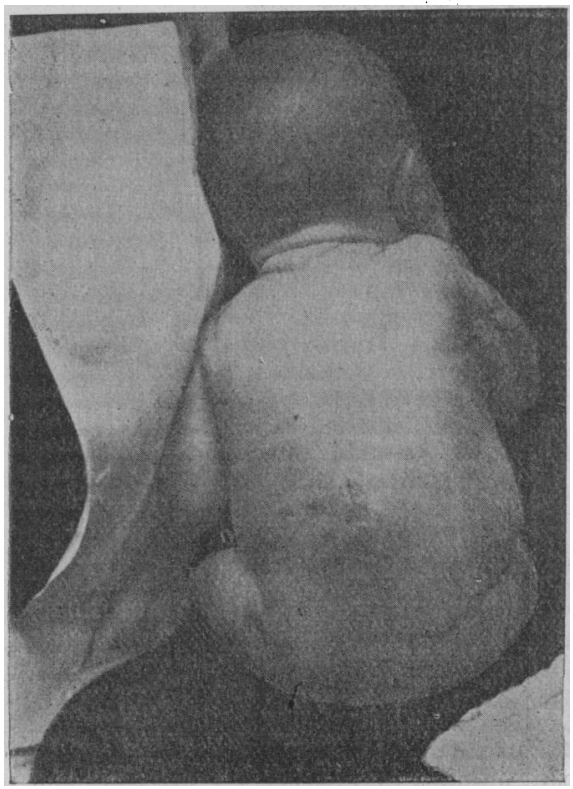

Fig. 4.-B. B., aged to weeks, or six weeks after excision of the spina

Into the general question of the operative treatment of spina bifida it is not necessary here to enter. In two former papers, one based on an experience of forty-six cases treated, I have dealt fully with certain aspects of it. With'reference to 
treatment, cases of spina bifida may be broadly classified as follows :

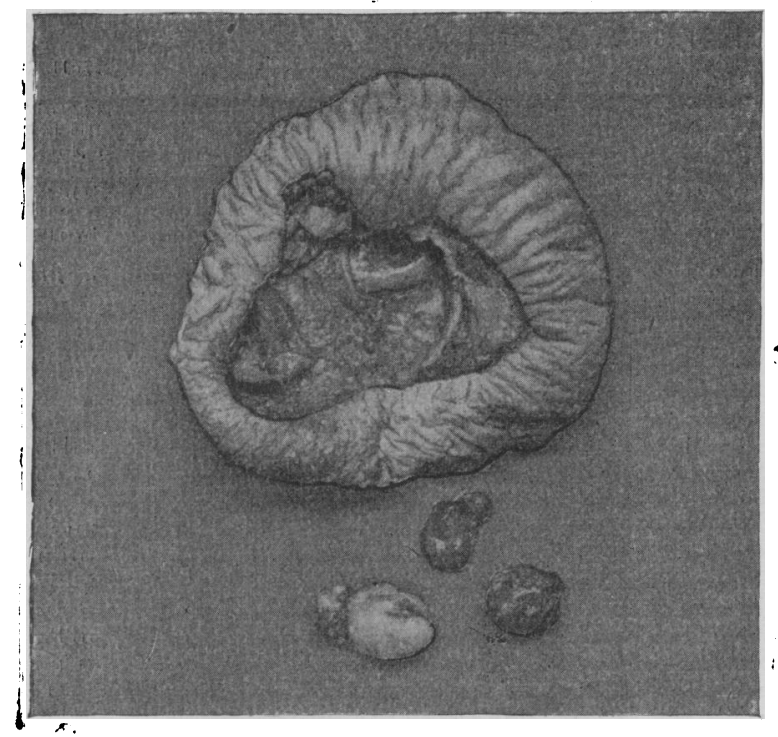

Fig. 5.-Excised spina bifida sac, which contained nerve trunks and plexiform neuromata; three of the latter are shown beside the sac. (Case of B. B.)

I. Cases with badly ulcerating or leaking sacs. These, as a rule, die from septic meningitis, whether subjected to treatment or not. I have, however, seen at least two such cases undergo complete cure by shrinkage and cicatrization of the sac, and have had several cases of recovery after prompt excision of a sac ruptured in parturition.

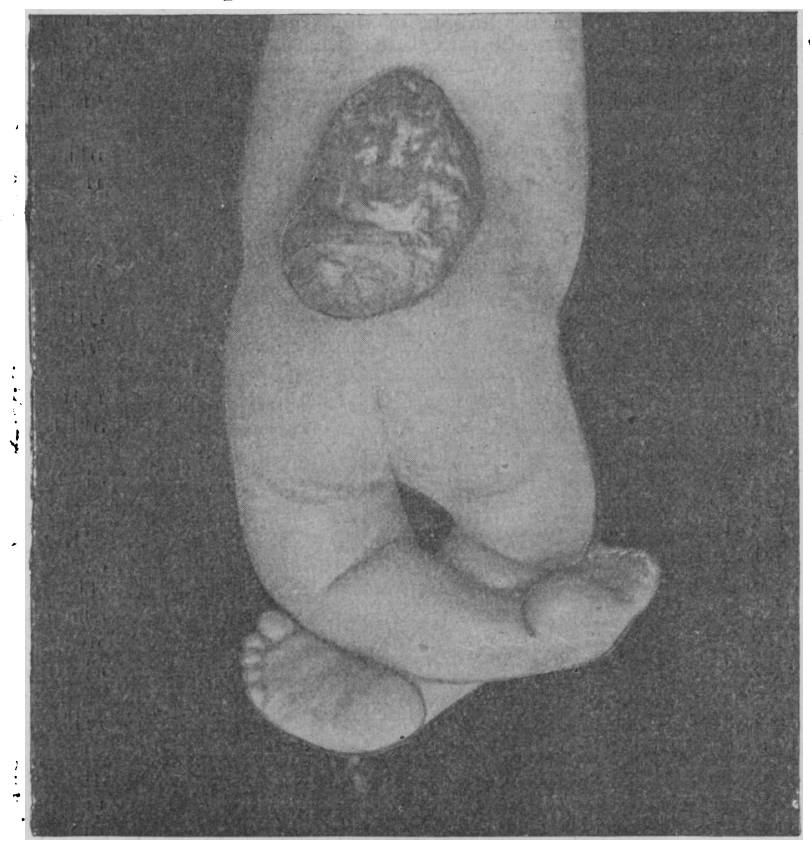

Fig. 6.-Case unsuited for excision, sac extensive and sessile, "with adherent spinal cord. Lower limbs rigid, and double club-foot present. Patient treated by injection by the method described.

2. Cases such as Fig. 6, in which the sac is so extensive and so sessile as to render it impossible to obtain flaps sufficiently free to effectually close the large gap. Such cases may be treated by the following methods: (a) Injection of Morton's fluid or other irritant, the syringe cannula being inserted through sound skin at least one inch clear of the margin of the tumour, and pushed through the muscles and fascia into the sac, thus avoiding subsequent leakage and permitting of the case being treated as an out-patient; (b) introduction of a strip of iodoform or other packing by the same route, the end of the strip being fixed by suture in the muscles and the skin closed by suture over it, and reopened after a week for its withdrawal ; (c) introduction of a needle for the purpose of tearing and roughening the sac. This method has a limited application in practice.

3. Cases in which the sac contains nerves or spinal cord, but is sufficiently pedunculated to permit of excision. Flaps of skin and flaps of mesoblastic tissues (muscle and fascia-the inclusion of bone is quite unnecessary) are fashioned and raised, and the sac is opened. Should the spinal cord be present all free sac is cut away, and the remainder sutured over the exposed cord and covered in by the flaps of fascia and skin. Where the filum terminale of the cord is present it may sometimes be freed and removed. with the sac. ${ }^{2}$

In cases where the sac contains nerves, the methods $I$ have followed have been mainly two: ( 1 ) In certain cases it is possible to dissect the nerve cords off the sac, place them in the spinal canal, suture the sac over them, closing in the canal, and covering all with the fascial and skin flaps. (2) Where the nerve strands are numerous this is impossible and the following plan has proved useful: Raise skin flaps and fascial flaps as usual, open the eac, and explore the interior; excise any portion free from nerve tissue, cut the remainder into ribbons by incisions made from the interior, avoiding the nerve tissue; gently but thoroughly roughen the entire interior-including the surface of the nerve bandswith the point of the knife. This has proved efficient in cases where no part of the sac could be either excised or cut into ribbons. ${ }^{3}$ Place the emptied and roughened or cut-up sac in the patent spinal canal, and cover in with the fascial and skin flaps.

The following modification of this method has also proved useful: Pack the "slashed" and roughened sac with iodoform or other sterile gauze, and partially suture. At the end of two or three days remove the packing, and complete the operation in the usual way.

The local result of such operation is, necessarily, less satisfactory than that obtained in cases where the conditions permit of a complete excision of the sac. The mortality also is somewhat higher. In two cases paralysis - of a lower limb in one, and of bladder and rectum in the other-has resulted in my hands. On the other hand, the operation has in several cases resulted in the relief of pre-existing paralysis. One is quoted above (Dr. Ross's case), and I have recorded others.

4. Cases of pure meningocele. The sac is simply opened, 4. Cases of pure mat away. Its neck is closed by ligature or suture, the method depending on the size of the aperture, and covered in by the fascial and skin flaps. Excision in anch cases constitutes an pasily accomplished radical cure.

In the treatment of hydrocephalus I have employed several methods described in the papers quoted. Of their comparative value I liave not yet been able to form an opinion sufficiently decided for publication. They are briefly the following :

a. Drainage of cerebro-spinal fluid at the time of operating or spina bifida. Into the vexed question of the route or routes hy which hydrocephalic fluid reaches the sac of a spina bifida it is not necessary to enter here. It is sufficient to restate the fact that in certain cases of spina bifida with hydrocephalus it is possible, by raising the child's head and shoulders after the spina bifida sac has been opened, to drain away gs much fluid as may be deemed safe, the amount being judged by the tension of the fontanelle.

$b$. Continuous drainage by a tube inserted into the spinal canal through the neck of an excised spina bifida sac at the time of operation. In several cases I have kept such a tube draining fluid inte the dressings for a week.

$c$. The establishing of a drainage channel from the spinal canal (at the site of and during the operation for, spina bifida) into either the peritoneal cavity or the cellular subcutaneous tissue, or both. This may be done by a drain which is absorbable after a time, or by a drain which is removed by secondary operation after a reriod of weeks or months. 
d. Drainage of the cerebral ventricles, either externally or into the meninges.

e. Application of various solutions (chiefly iodine) to the interior of the cerebral ventricles. What experience I have had of this method has impressed me favourably. It is not, however, devoid of risk. The amount and the strength of an injection will depend on the degree of dilution likely to occur internally, that is, on what the operator knows, from previous drainage or otherwise, of the amount of fluid present. In any case, for a first injection something less potent than Morton's fluid should be employed.

To those familiar with the literature of the subject it will be evident that certain of these methods of attempted treatment of hydrocephalus have been already brought forward by others. With reference to method (c), since I published it, in July, I898, I observe that it has been independently published by another surgeon, and may have been so previously. My experience of its effects has not been sufficiently extensive to warrant my putting it forward in other than a tentative way.

Exception has been taken by more than one critic to my formerly-published opinion that "open operation " (that is, excision of spina bifida) "in suitable cases has a mortality little, if at all, higher than has the operation for the radical cure of inguinal hernia." That opinion I believe to be well founded.

The two cases detailed above were treated throughout as out-patients at the Glasgow Children's Hospital ; and, without at present submitting a statement of all cases of spina bifida treated by excision, or of all cases of spina bifida and hydrencephalocele treated as out-patients after excision or injection, it may be well to submit here a statement of the cases of spina bifida which I have treated as out-patients after excision at the Glasgow Children's Hospital. These, I find, have numbered nine in all. One of the nine, however, was treated at the time in which I had made it a rule to attempt operative cure in all cases of spina bifida without exception. It was brought, aged I week, in January, 1898. Obviously very ill, with temperature $100.4^{\circ}$, the infant had a collapsed and sloughing spina bifida in the dorsal region which was said to have ruptured during birth. This I at once resected under chloroform. The child died on the sixth day after operation, having developed an eruption which was pronounced by a medical man to be chicken-pox, but which, to my mind, was probably due to pyaemia or septicaemia. It is evident that this case with its sloughing sac and developing meningitis should not be placed with the others. Of the 8 cases which were non-septic at the time of operation, 6 are at present alive and well; I (J. B.) died as the result of operative preparations; and $\mathrm{I}(\mathrm{H}$. $\mathrm{H}$ ) died of whoopingcough three years after operation. The subjoined is the complete list :

Cases of Excision of Spina Bifida; Treated after Operation as Out-patients at the Children's Hospital, Glasgow.

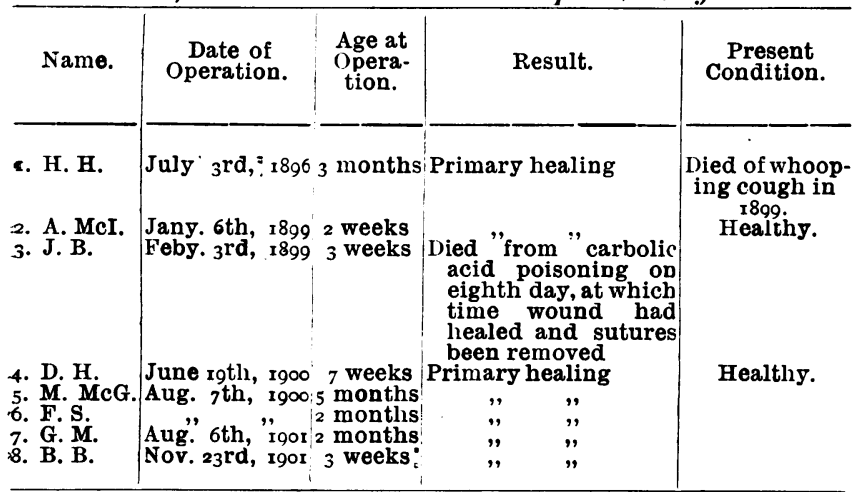

NoTE.-Since the foregoing cases were tabulated two further cases (both meningoceles) have been treated as out-patients after excision at the Children's Hospital, Glasgow. Both healed by first intention.

Six of the cases were pure meningoceles. Of these, Case II was one of the largest spina bifida sacs I have seen. The parents were afraid to lift the child for fear of the sac " tearing away," and it was so extensive and thin that I felt constrained to remove it then and there, and did so.
Case vur contained nerve cords and neuromata, and is detailed above. Case Iv, which was a cervical sac, presented the peculiarity that the neck of the sac felt solid, and gentle pinching of the solid part produced smart jerking upward of the lower limbs, after the manner of the wooden figure toy on pulling the string. At the operation the solid portion was found to be a geniculate bend of the spinal cord into the neck of the sac, to cover which specially large flaps had to be dissected up. For a time after operation the jerking of the legs could be excited at the observer's will, but of late the coverings have becone more tough. The child has been late in walking, but is now, at the age of nearly two years, commencing to walk, apparently normally (so far as can yet be judged), and is otherwise well.

Case III died of acute gastro-enteritis, produced, apparently, by absorption of carbolic acid. Carbolic acid poisoning has occurred in several of the cases of spina bifida I have seen though in this one alone with fatal effect. The case is mentioned in the paper of $1899,{ }^{4}$ and the following is the note there:

"On the morning of the operation the child was found to be suffering from carbolic acid poisoning. Its urine was blackish-green when passed, and the child was markedly collapsed, and vomited frequently. In three cases I have seen such symptoms follow the application of carbolic dressings to spina bifida sacs prior to operation; and it seems nct improbable that the very thin parietes, bathed on one side by serous fluid, offer conditions under which absorption f:om the outside may occur with great facility. On the two form er occasions on which symptoms of carbolic poisoning were present I deferred operation. On this occasion the child appeared so ill that I thought it better to proceed with the operation in the hope that the evacuation of the contents cf the sac might lead to relief of the symptoms. Unfortunately this did not occur. The symptoms persisted, and were aggravated by increase of the vomiting and diarrhoea, with evidence of acute gastro-intestinal irritation, accompanied by subnormal temperatures. The wound, in spite of the child's increasing weakness, healed before death occurred from exhaustion on the eighth day."

Since the date of the foregoing I have seen toxic effects of carbolic acid in several further cases. Indeed, Case vilI was an instance in point. The urine here was blackish-green for three days after operation. In this case no dressing was applied beforehand; the parts were washed, cleansed with turpentine and spirit, and merely sponged with I in 40 carbolic lotion just prior to operation. The instruments used were placed in $I$ in 60 carbolic lotion, and the dressing was cyanide gauze sparingly moistened with $I$ in 40 carbolic lotion. The entire quantity of carbolic acid to be absorbed had been purposely reduced to a minimum, but proved sufficient to affect the urine.

In reviewing a somewhat extensive list of cases of spina bifida, hydrocephalus, and hydrencephalocele treated by operation in hospital and private practice, I am conscious of a growing belief that infants of tender age operated on for these and various other surgical affections fare at least as well in the care of their mothers as in the wards of a hospital, however well-conducted and efficient in the treatment of older beings.

REFERENCES

1 BRITISH MEdical JoURNaL, October 15th, 1898, and Glasgow Hospital Reports, vol. ii. 1899. 2 Glasgow Hospital Reports, vol. ii. 3 BRITISH MEDICAL JOURNAL, October $x_{5}$ th, 1898 (7 cases since). 4 Glasgow Hospital Reports, vol. ii.

\section{(200rts, vol.}

\section{MEDICAL, SURGICAL, OBSTETRICAL, THERA- PEUTICAL, PATHOLOGICAL, ETc.}

\section{ON THE EMPLOYMENT OF ADRENALIN AS AN ADJUNCT TO THE LIGHT TREATMENT \\ OF LUPUS.}

WHEN Dr. Takamine delivered his address on adrenalin in Edinburgh, and demonstrated its singular power of blanching a mucous surface, it occurred to me that this property might be utilized in the light treatment of lupus, seeing that the 